\title{
Prevalence of refractive error among school children in the Cape Coast Municipality, Ghana
}

\section{G O Ovenseri-Ogbomo' $\checkmark$ O Omuemu ${ }^{2}$}

'Department of Optometry, University of Cape Coast, Ghana; ${ }^{2}$ Department of Community Health, College of Medicine, University of Benin, Nigera
Correspondence: Ovenseri-Ogbomo Department of Optometry, University of Cape Coast, Ghana

Tel +233243462 912

Email ovenseriogbomo@yahoo.com
This article was published in the following Dove Press journal:

Clinical Optometry

3 July 2010

Number of times this article has been viewed

\begin{abstract}
Uncorrected refractive errors continue to remain a public health problem among different population groups. Among school children, it has a considerable impact on learning and academic achievement especially in under-served and under-resourced communities. There is a dearth of information about the magnitude of the problem in Ghana. A school based crosssectional study was carried out to estimate the prevalence and distribution of refractive error among school children in the Cape Coast Municipality of Central Region of Ghana. A total of 1103 school children were enumerated out of which 961 underwent a full eye examination. The children were aged between five and 19 years (mean $=10.5 \pm 3.4$ years, $95 \%$ confidence interval [CI]: 10.3-10.7). Cycloplegic refraction was performed on each child who failed the reading test. Hyperopia was defined as spherical power of $\geq+2.00$ diopters sphere (DS), myopia as $\leq-0.50 \mathrm{D}$ and astigmatism as a cylindrical power of $\leq-0.50 \mathrm{D}$. Of the children examined, only $0.6 \%$ had previously had an eye examination. The prevalence of low vision and blindness in the study population was $0.9 \%$ (95\% CI: $0.881-0.919)$ and $0.1 \%$ (95\% CI: $0.081-0.119)$ respectively. $25.6 \%(95 \% \mathrm{CI}: 22.84-28.37)$ of the children examined had refractive errors. This comprises of 44 (4.6\%; 95\% CI: 3.3-5.9) hyperopia, 66 (6.9\%; 95\% CI: 5.3-8.5) myopia and $135(14.1 \%$; 95\% CI: 11.9-16.3) astigmatism of the 957 children examined. The study concludes that uncorrected refractive error is a common cause of visual impairment among school children in the municipality. A low uptake of eye care is also noted in the study. The study therefore recommends the education authority, in collaboration with the District Health Directorate, institutes appropriate measures to ensure compulsory eye examination for school children in the Cape Coast Municipality.
\end{abstract}

Keywords: refractive error, school children, Ghana, visual impairment, uptake, socioeconomic status

\section{Introduction}

Visual impairment resulting from uncorrected refractive errors remain a significant public health problem worldwide. The significance of refractive error as a cause of visual impairment only recently assumed ascendancy following the re-conceptualization of visual impairment (blindness and low vision) using presenting visual acuity rather than best corrected visual acuity. ${ }^{1}$ Using presenting visual acuity (VA) allows for the estimation of the magnitude of visual impairment due to refractive errors. Blindness is defined in terms of visual acuity (VA) as $<3 / 60$ in the better seeing eye and low vision as VA between $6 / 18$ to $3 / 60$ in the better seeing eye. ${ }^{2}$

An estimated 2.3 billion people worldwide have a refractive error and of these, only 1.8 billion have access to an eye examination and affordable refractive correction. ${ }^{3}$ The majority of the 500 million who do not have access to refractive error services live in 
developing countries and are mainly children. ${ }^{3}$ Undetected and uncorrected refractive errors are a particularly significant problem in school children. ${ }^{4}$ Poor vision and an inability to read material on the chalkboard due to refractive error can profoundly affect a child's participation and learning in the classroom. ${ }^{4}$ It also has serious social implications for the child in school. Teachers who do not realize the plight of the child with respect to his/her visual status may unwittingly scold the child for being lazy and stupid and humiliate him/ her persistently. Unsympathetic schoolmates also taunt the child in the classroom as well as in the playground. These factors may combine to make the child drop out of school and be a victim of the attendant social problems associated with school drop-outs. Not infrequently, parents and siblings may undermine and discourage these unfortunate children. One report has documented the severe impact of poor vision on primary school children in Brazil. ${ }^{5}$ Specifically, the authors found that children with reduced vision had a $10 \%$ higher probability of dropping out of school, an $18 \%$ higher probability of repeating a grade and scored about 0.2 to 0.3 standard deviations lower on achievement test. This study did not, however, indicate whether the poor vision was due to refractive error or not. It does provide a peek into the impact of poor vision on academic achievement. ${ }^{5}$

To further understand the epidemiology of refractive errors among school children a uniform protocol has been developed. ${ }^{4}$ Several studies have been conducted in different parts of the world using this protocol. ${ }^{6-11}$ The latest global estimates of visual impairment suggests that among children aged 5-15 years, 12.8 million were visually impaired due to refractive errors representing a prevalence of $0.97 \%$ with higher prevalence reported in China and urban areas of south east Asia. ${ }^{12}$

This study was therefore undertaken to determine the magnitude of refractive errors among school children in the Cape Coast Municipality of Ghana.

\section{Method}

\section{Sampling}

Cape Coast is the administrative capital of Central Region, one of the 10 administrative regions of Ghana. With an estimated population of 1.6 million, Central Region has an annual growth rate of $2.1 \%$ and a population density of 162.2 persons per square kilometer. ${ }^{13}$

A cross-sectional descriptive study was carried out to study the magnitude of refractive errors among school children in the municipality. The basic schools in Cape Coast Municipality are divided into six circuits. Each circuit constitutes a cluster of schools from which one school was randomly selected. The study involved the examination of all school children in the schools that were selected at random from each circuit. Using the expression $n=Z^{2}(1-p)(p) / b^{2}$ (where $\mathrm{n}=$ minimum sample size, $\mathrm{p}=$ anticipated prevalence [assumed to be 50\%], b = desired error bound taken as 5\% and $\mathrm{Z}=$ the standard score at $95 \%$ ), a minimum sample of 384 was calculated. To account for a $10 \%$ attrition rate, the sample size became 423. To accommodate for inefficiencies associated with cluster sample design, an increase of $100 \%$ is allowed ${ }^{14}$ due to the small number of clusters. This gave a final sample size of 846 . In the present study, 1103 children were enumerated. Of these, 961 children participated and were examined giving a participation rate of $87 \%$.

\section{Field operation}

Institutional approval to carry out the study was obtained from the Department of Optometry, University of Cape Coast for the study. Additionally, permission was sought and obtained from the local education authority. The heads of selected schools were briefed on the purpose of the study and they signed an informed consent form on behalf of the school children. In addition, the assent of children was sought before they were examined.

The examination team consisted of two optometrists and five sixth year clinical students. The two optometrists performed all the refraction and cross-checked the examination form to ensure that they were accurately filled out. Before the field operation, the clinical team were briefed on the purpose of the study and were trained on carrying out clinical procedures in the field. All the clinical students had previously participated in out of clinic eye care programs.

\section{Clinical examination}

The original Refractive Error Study in School Children (RESC) protocol was relied upon in designing the examination form. VA was measured at a distance of $6 \mathrm{~m}$ using the Snellen tumbling E chart. Children who wore glasses also had their VA taken while wearing their glasses. The presence of ocular deviation (phoria and tropia) was determined using the cover test. Ocular health examination (including internal and external) was performed for all children examined. Subjective refraction followed by cycloplegic refraction using cyclopentolate hydrochloride (1.0\% solution) was carried out for all children. We performed retinoscopy 30 minutes after the instillation of the cyclopentolate hydrochloride. The final prescription and the best-corrected VA were recorded. Medication was given for minor ocular conditions at no cost at the time 
of the examination while those who needed further medical or diagnostic work-up were referred to hospitals in the area.

The children with presenting VA $=6 / 12$ or worse in the better seeing eye whose vision improved with refraction were provided with glasses at no cost.

\section{Data management and analysis}

The data forms were checked for accuracy and completeness in the field before data entry. Refractive error was assigned as the cause of the visual impairment if in the absence of any obvious pathology, vision improved to $6 / 6$ or better with refraction. Amblyopia was assigned the cause of visual impairment when, in the absence of any noticeable pathology or abnormality, there was no improvement in vision with refraction.

Hyperopia was defined as a spherical power of $\geq+2.00$ diopters sphere (DS) in both eyes or in one eye (if the other eye is emmetropic). Myopia was defined as a spherical power of $\leq-0.50 \mathrm{DS}$ in both eyes or in one eye (if the other eye was emmetropic). A cylindrical power of $\leq-0.50$ diopters cylinder (DC) in both eyes or in one eye (if the other eye was emmetropic) was considered as astigmatism. When there was a difference in refraction between the two eyes greater than 2.00 diopters (D), it was designated as anisometropia. The results of the right eye were used to determine the refractive errors present. This is because the mean refractive error measurement has been reported to be similar in both left and right eyes. ${ }^{6}$

The socioeconomic status (SES) of the children was inferred from the occupation of their parents. As a proxy for estimating the SES of children, parents' education, occupation and employment status have been employed. ${ }^{15}$ Professional occupations like engineering, accounting, lawyers etc were considered as high SES; occupations within government sectors which are not professional occupations such as civil servants, police and teachers were categorized as medium SES. Occupations like trading, farming, artisans and the like were categorized as low SES. The occupation of both parents was examined in assigning a child to a SES category or of either parent depending on which parent is higher.

The data sheets were collected and the data was entered for computer analysis. The Statistical Package for Social Sciences (version 12; SPSS Inc., Chicago, IL) was used for data analysis as well as Microsoft Excel (2007; Microsoft, Redmond, WA).

\section{Results \\ Study population}

A total of 1103 school children were enumerated for the study out of which 961 children were examined. This gave
Table I Distribution of enumerated, examined and valid results by gender

\begin{tabular}{llll}
\hline & Enumerated (\%) & Examined (\%) & Valid results (\%) \\
\hline Males & $439(39.8)$ & $297(30.9)$ & $297(31)$ \\
Females & $667(60.2)$ & $664(69.1)$ & $660(69)$ \\
Total & $1103(100)$ & $961(100)$ & $957(100)$ \\
\hline
\end{tabular}

a participation rate of $87.1 \%$. The nonresponders include children who were absent on the day of the examination and those who probably were scared of the test and declined to participate. Of the 961 children that were examined, the results of four children were excluded from the analysis because their examination forms were not completely filled out. The results of 957 children are presented below (Table 1).

The subjects consist of 660 females (69\%) and 297 males (31\%). The children were aged 5-19 years (mean $=10.5 \pm 3.4$ years, 95\% confidence interval $[\mathrm{CI}]$ : $10.3-10.7)$. The majority were aged $8-13$ years $(52.9 \%)$. The modal age group for the males was 5-7 years while for the female it was 11-13 years. The mean age for the females was $10.7 \pm 3.2$ years (95\% CI: 10.4-10.9), while that for the males was $10.2 \pm 3.7$ years (95\% CI: 9.7-10.6). There was a significant difference between the mean age of male and female children $(\mathrm{t}=2.049, P=0.04)$.

The majority of the children examined were children whose parents are in the low SES group. They accounted for $52.7 \%$ followed by those in the medium SES with $36.7 \%$ and those in the high SES with $10.6 \%$ (Table 2). The mean ages of children in the different SES levels were 10.0 years (95\% CI: 9.4-10.6, $\mathrm{SD}=3.13)$, 10.1 years (95\% CI: 9.8-10.5, $\mathrm{SD}=3.27)$ and 10.9 years $(95 \% \mathrm{CI}: 10.6-11.2 \mathrm{SD}=3.45)$ for high, medium and low SES respectively. This difference in mean was statistically significant $(\mathrm{F}=5.974, P=0.003)$.

The study further revealed that of the 957 children examined, only six (two males and four females) reported having previously had an eye examination and were wearing their glasses. This represents a meager $0.6 \%$ of the children examined. This comprised of three children in the low SES, two and one child respectively in the high and medium SES.

Table 2 Distribution of parent's SES with gender

\begin{tabular}{llll}
\hline Socio-economic status & Sex & \multicolumn{2}{c}{ Total (\%) } \\
\cline { 2 - 3 } & Male (\%) & Female (\%) & \\
\hline High & $2 \mathrm{I}(7 . \mathrm{I})$ & $80(12 . \mathrm{I})$ & $\mathrm{I0I}(\mathrm{I0.6})$ \\
Medium & $99(33.3)$ & $253(38.3)$ & $352(36.7)$ \\
Low & $177(59.6)$ & $327(49.6)$ & $504(52.7)$ \\
Total & $\mathbf{2 9 7 ( 1 0 0 )}$ & $\mathbf{6 6 0 ( 1 0 0 )}$ & $\mathbf{9 5 7 ( 1 0 0 )}$ \\
\hline
\end{tabular}

Notes: $\chi^{2}=10.271, \mathrm{df}=2, P=0.006$. 
Whether a child had had an eye examination was not related to the parent's $\operatorname{SES}\left(\chi^{2}=3.641, P=0.162\right)$.

\section{Presenting visual acuity}

The distribution of VA for each eye is presented in Table 3. Sixty-six $(6.9 \%)$ had mild visual impairment ${ }^{2}$ (VA between $6 / 9$ and 6/12) in the right eye while 63 (6.6\%) had mild visual impairment in the left eye. Using the International Classification of Diseases (ICD) definition, ${ }^{2}$ nine $(0.9 \%)$ and $13(1.4 \%$ ) had low vision (VA $<6 / 18$ to $3 / 60$, that is visual impairment category $\mathrm{I}$ and $\mathrm{II}^{2}$ ) in the right and left eye respectively.

With blindness and low vision defined using vision in the better seeing eye, the prevalence of blindness and low vision in the study population was $0.1 \%(95 \% \mathrm{CI}: 0.081-0.119)$ and 0.9\% (95\% CI: 0.881-0.919) respectively. In $69(7.2 \%)$ of the children examined, VA could not be determined due to lack of cooperation from the child.

Of the six children who had previously had an eye examination, two had a $\mathrm{VA}=6 / 6$. Three had a $\mathrm{VA}=6 / 9$ while one had a $\mathrm{VA}=6 / 24$

\section{Refractive errors}

Both subjective and objective refractions were performed for all the children examined. The results were used to identify the various types of refractive errors presented. In three $(0.3 \%)$ of the children, refraction could not be performed due to corneal abnormality. The results of the right eye were used to determine the refractive errors present. ${ }^{6}$ The distribution of the type of refractive errors by gender and age is presented in Tables 4 and 5.

Table 4 shows that 245 children representing $25.6 \%$ (95\% CI: $22.84-28.37 \%$ ) of the children examined had refractive errors. This comprises of $44(4.6 \% ; 95 \%$ CI: 3.3-5.9) hyperopia, 66 (6.9\%; 95\% CI: 5.3-8.5) myopia and 135 (14.1\%; 95\% CI: 11.9-16.3) astigmatism

Table 3 Distribution of visual acuity

\begin{tabular}{lll}
\hline Visual acuity & Right eye (\%) & Left eye (\%) \\
\hline$>6 / 6$ & $812(84.8)$ & $811(84.7)$ \\
$6 / 9$ & $55(5.7)$ & $51(5.3)$ \\
$6 / 12$ & $11(1.1)$ & $12(1.3)$ \\
$6 / 18$ & $1(0.1)$ & $6(0.6)$ \\
$6 / 24$ & $3(0.3)$ & $2(0.2)$ \\
$6 / 36$ & $2(0.2)$ & $3(0.3)$ \\
$6 / 60$ & $3(0.3)$ & $2(0.2)$ \\
$3 / 60$ & $1(0.1)$ & $1(0.1)$ \\
Undetermined & $69(7.2)$ & $69(7.2)$ \\
Total & $957(100)$ & $957(100)$ \\
\hline
\end{tabular}

Table 4 Distribution of refractive errors by gender

\begin{tabular}{llll}
\hline $\begin{array}{l}\text { Type of refractive } \\
\text { error }\end{array}$ & Sex & Total (\%) \\
\cline { 2 - 3 } & Male (\%) & Female (\%) & \\
\hline Emmetropia & $226(76.1)$ & $483(73.2)$ & $709(74.1)$ \\
Hyperopia & $8(2.7)$ & $36(5.5)$ & $44(4.6)$ \\
Myopia & $22(7.4)$ & $44(6.7)$ & $66(6.9)$ \\
Astigmatism & $4 I(13.8)$ & $94(14.2)$ & $135(14.1)$ \\
Undetermined & $0(0.0)$ & $3(0.5)$ & $3(0.3)$ \\
Total & $\mathbf{2 9 7}(\mathbf{1 0 0 . 0})$ & $\mathbf{6 6 0 ( 1 0 0 . 0 )}$ & $\mathbf{9 5 7}(\mathbf{1 0 0 . 0})$ \\
\hline
\end{tabular}

Notes: $\chi^{2}=5.171, \mathrm{df}=4, P=0.270$.

of the 957 children examined. Thus of the 245 children with refractive errors, the commonest refractive error was astigmatism accounting for 55\% followed by myopia and hyperopia with $27 \%$ and $18 \%$ respectively. The type of refractive error was dependent on the age of a child $\left(\chi^{2}=46.17, \mathrm{df}=16, P=0.000\right)$.

Table 5 shows a decline in hyperopia from $10.4 \%$ among children aged five-seven years to $0 \%$ in the age group 17-19 years, while myopia increased from $0.9 \%$ in the age group five-seven years to $12.5 \%$ in the age group 17-19 years.

The distribution of type of refractive error with SES is show in Figure 1 below.

The maximum degree of refractive errors found in the study were +7.00 DS for hyperopia, $-2.50 \mathrm{DS}$ for myopia and $-6.25 \mathrm{DC}$ for astigmatism. All these were previously undiagnosed and uncorrected.

Spherical equivalent was computed using the refractive errors of the right eye. The mean spherical equivalent for the subjects was $+0.56 \mathrm{DS}(95 \% \mathrm{CI}: 0.50-0.62, \mathrm{SD}=+0.97$ $\mathrm{DS}$, range $=-9.63 \mathrm{DS}-+7.00 \mathrm{DS})$. In terms of gender, the mean spherical equivalent for the male was +0.54DS $(95 \%$ CI: $0.46-0.62)$ while that of the female was $+0.57 \mathrm{DS}(95 \%$ CI: $0.49-0.65)$. There was no statistical difference between the spherical equivalent for both male and female subjects ( $\mathrm{t}=-0.452, P=0.651)$.

Similarly, the mean spherical equivalent for the types of refractive errors was computed. The mean spherical equivalent for hyperopia was $+2.55 \mathrm{DS}$ (95\% CI: 2.20-2.90). The mean spherical equivalent for myopia was $-0.88 \mathrm{DS}(95 \%$ CI: $-1.17--0.60)$ while that for astigmatism was $+0.23 \mathrm{DC}$ (95\% CI: 0.00-0.46). Figure 2 shows the histogram of the distribution of right eye spherical equivalent.

The spherical equivalent shows a slight negative correlation with the age of the subjects $(\mathrm{r}=-0.320, P=0.000)$. This indicates that as a child grows, the refractive state tends towards less hyperopia and more myopia all other factors being equal. 
Table 5 Distribution of refractive error by age

\begin{tabular}{|c|c|c|c|c|c|}
\hline \multirow[t]{2}{*}{ Age (yrs) } & \multicolumn{5}{|c|}{ Type of refractive error } \\
\hline & Emmetropia & Hyperopia & Myopia & Astigmatism & Undetermined \\
\hline $5-7$ & I 73 (74.9) & $24(10.4)$ & $2(0.9)$ & $30(13.0)$ & $2(0.9)$ \\
\hline $8-10$ & $186(73.2)$ & $10(3.9)$ & $21(8.3)$ & $36(14.2)$ & I $(0.4)$ \\
\hline $11-13$ & $193(76.3)$ & $4(1.6)$ & $23(9.1)$ & $33(13.0)$ & $0(0.0)$ \\
\hline $14-16$ & 145 (7I.4) & $6(3.0)$ & $18(8.9)$ & 34 (16.7) & $0(0.0)$ \\
\hline $17-19$ & $12(75.0)$ & $0(0.0)$ & $2(12.5)$ & $2(12.5)$ & $0(0.0)$ \\
\hline Total & 709 (74.1) & $44(4.6)$ & $66(6.9)$ & $135(14.1)$ & $3(0.3)$ \\
\hline
\end{tabular}

Notes: $\chi^{2}=46.170, \mathrm{df}=16, P=0.000$.

\section{Discussion}

Childhood blindness is one of the priority conditions targeted in VISION 2020: The Right to Sight Initiative of the World Health Organisation. ${ }^{16}$ Uncorrected refractive errors and other visually impairing conditions in school children can hinder education, personality development and career opportunities. Additionally, they can impose significant economic burden on the family and society. There is therefore the need for refractive errors to be detected and spectacle correction promptly provided to mitigate these problems.
Knowledge of the prevalence of refractive errors among school children in the Cape Coast Municipality can help relevant authorities plan and provide eye care in the municipality. This study attempts to provide this information.

In this study cycloplegic refraction was performed for the school children. This is different from the noncycloplegic refraction used by Mabaso et a $\mathrm{l}^{17}$ and Adegbehingbe et al ${ }^{18}$ in their respective studies of school children in South Africa and Nigeria. Many studies on the subject have also used cycloplegic refraction in determining the refractive status

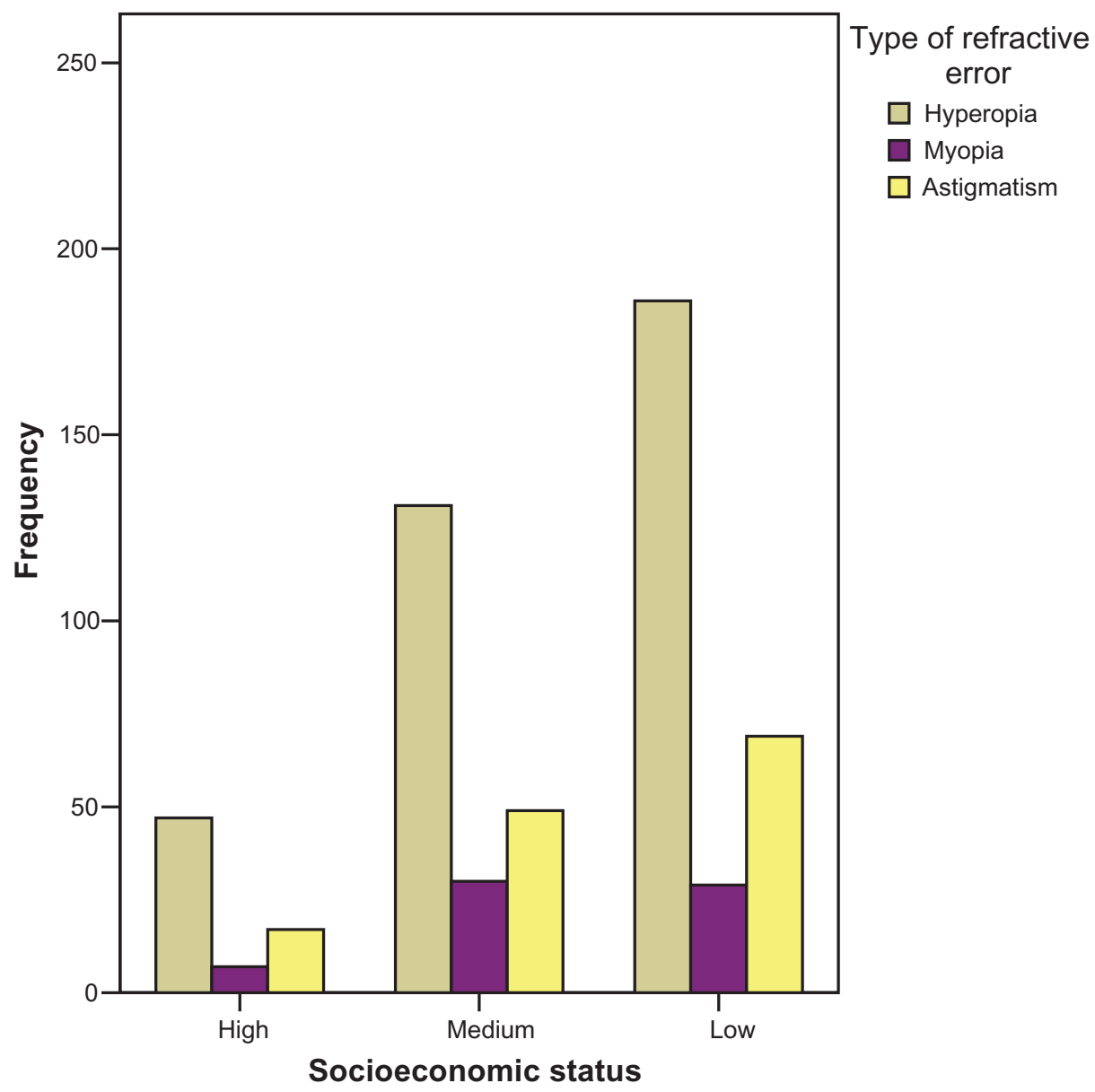

Figure I Distribution of the type of refractive error with parent's socioeconomic status. 


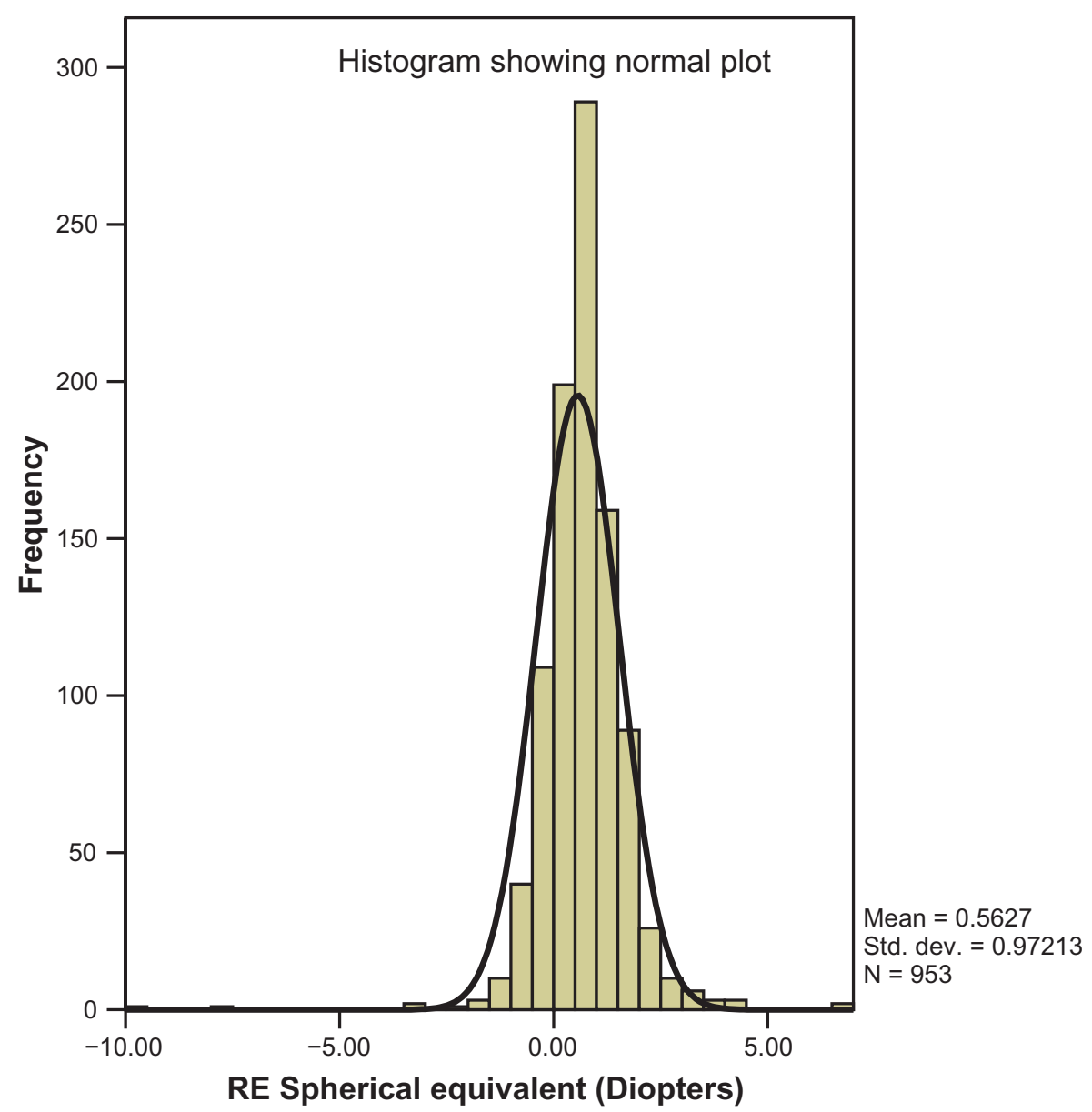

Figure 2 Histogram showing the distribution of spherical equivalent of the right eye.

of school children. ${ }^{7-11}$ Using cycloplegic refraction tends to reveal otherwise latent hyperopia especially in children with a high accommodative amplitude. This therefore leads to higher proportion of hyperopia and hence the prevalence of refractive error.

This high proportion of females in the study was because one of the schools surveyed is an all-female school. It does not reflect the gender enrolment in the municipality. In general, there has been a considerable increase in school enrolment following the introduction of the school feeding program by the government of Ghana.

The overall prevalence of blindness (VA $<3 / 36$ ) and low vision (VA $<6 / 18-3 / 36$ ) was $0.1 \%$ and $0.9 \%$ respectively. This was similar to $0.17 \%$ reported by Dandona \& Dandona for a population-based study in India. ${ }^{19}$ This similarity should be taken with caution as both studies involved children in different settings. In the study, ${ }^{19}$ blindness was defined as $\mathrm{VA}<6 / 60$. It is difficult to compare the prevalence of blindness and low vision in this study with other studies involving school children. This is because while the prevalence in this study is based on the ICD definition of blindness and low vision, other studies ${ }^{6,7,9,17,20}$ computed the prevalence of VA of 20/40 (6/12) or worse.

The proportion of children who had previously had an eye examination in this study $(0.6 \%)$ is comparable to $0.58 \%$ reported for rural India ${ }^{6}$ but slightly more than the $0.3 \%$ reported for school children in Agona District of the Central Region of Ghana ${ }^{21}$. It is considerably less than $30.3 \%$ among students in Tanzania, ${ }^{22}, 42.3 \%$ in Egypt ${ }^{15}$, and 2.7\% in South Africa. ${ }^{10}$ In a study of 1707 secondary school children in Ile-Ife, Nigeria, none of the children examined had previously had an eye examination. ${ }^{18}$ Because of the relative ease of obtaining refractive error services, low uptake of this service is said to reflect a lack of awareness and importance of accessing eye care services in any society. Factors responsible for poor uptake of refractive services are both social and economic. Wearing of spectacles is sometimes associated with blindness and individuals abhor the social stigmatization associated with blindness. On the economic front, refractive error services are expensive to procure. 
This is because refractive services in developing countries are still being provided as a business model rather than a service model.

Furthermore, the uptake of refractive error services as indicated by the proportion of children who had previously had an eye examination was not associated with socioeconomic status of the parents $(P=0.162)$. The failure to find any association between SES and uptake of refractive error services may be due to the small proportion of the children who had previously had an eye examination ( $0.6 \%$ ). Additionally, SES appear not to influence the distribution of the types of refractive error especially myopia. This may in part be due to the fact that the educational system in Ghana is fairly uniform at the basic school level. The curriculum is similar and requires about the same amount of near work from all school children.

Refractive error was identified in $25.6 \%$ of the subjects. This figure is low when compared to studies on comparable groups from other regions of the world. ${ }^{6-8,10,20,22}$ Interestingly, the prevalence of refractive errors in this study is comparable to the prevalence of $22.3 \%$ of uncorrected refractive errors found among 629 school children aged 12-16 years in Singapore. ${ }^{23}$ This is a rather curious finding as the Oriental population has been shown to have higher prevalence of refractive errors than any other population group. This similarity in the prevalence may be due to the different definition used in the two studies. In the Singapore study, uncorrected refractive error was defined as at least $0.2 \operatorname{logMAR}$ units over habitual VA. Besides, myopia was defined as a spherical equivalent of at least $-1.00 \mathrm{D}$, hyperopia as $+1.00 \mathrm{D}$ and astigmatism as $-1.00 \mathrm{D}$. Our finding is also similar to a prevalence of $22.1 \%$ among school children aged $7-15$ years in Cairo. ${ }^{15}$ It was, however, higher than the prevalence of $11.6 \%$ reported by Kawuma and Mayeku ${ }^{24}$ for school children in Kampala and $13.5 \%$ reported by Adegbehingbe et al ${ }^{18}$ among school children in Ile-Ife, Nigeria. Astigmatism was the most common form of refractive error present among those with refractive error $(55.0 \%)$. This was followed by myopia $(27.0 \%)$ and hyperopia (18.0\%). This pattern is consistent with findings from Kampala ${ }^{24}$ where astigmatism was most common, affecting $52 \%$ of those with refractive errors. The proportion of children who had myopia and hyperopia were higher compared to children in rural population of India. ${ }^{6}$ Though this study used the same criteria for defining myopia and hyperopia, this difference may be due to the different population. The prevalence of refractive error has been shown to vary for different regions of the world. ${ }^{12}$ The preponderance of astigmatism in this study would need to be further investigated.
The study also revealed that there was a negative correlation between spherical equivalent and the age of the children. This is consistent with other studies ${ }^{7-8}$ where there was a decrease in the proportion of hyperopia with age and an increase in myopia. This trend may be attributed to the theory of emmetropization in which there is a shift from hyperopia in early childhood to emmetropia as the child grows. Myopia has been reported to be associated with female gender, older children, parental educational attainment, ethnicity, urban dwellers and parental and sibling myopia. ${ }^{6,8,17}$

From our study, we can conclude that refractive error is a significant cause of visual impairment among school children in the Cape Coast Municipality. There is also a low uptake of refractive error services among this group. This may in part account for the high prevalence of refractive error. We therefore recommend that cost-effective strategies for vision screening of school children be incorporated into the school health program of the Ministry of Health and Education if the devastating impact of visual impairment on a child's education and development be prevented.

\section{Disclosure}

The authors report no conflicts of interest in this work.

\section{References}

1. Dandona R, Dandona L. Refractive error blindness. Bull World Health Organ. 2001;79(3):237-243.

2. World Health Organization. International Statistical Classification of Diseases and Related Health Problems. 10th Revision, 2nd Ed. Geneva, World Health Organization: 2005.

3. Holden BA, Sulaiman S, Knox K. The challenge of providing spectacles in the developing world. Community Eye Health. 2000;13(33): 9-10.

4. Negrel AD, Maul E, Pokharel GP, Zhao J, Ellwein LB. Refractive error study in children: sampling and measurement methods for a multicountry survey. Am J Ophthalmol. 2000;129(4):421-426.

5. Gomes-Neto J, Hanushek E, Leite R, Frota-Bezzera R. Health and schooling: evidence and policy implications for developing countries. Econ Edu Rev. 1997;16(3):271-282.

6. Dandona R, Dandona L, Srinivas M, et al. Refractive error in children in a rural population in India. Invest Ophthamol Vis Sci. 2002;43(3):615-622.

7. Maul E, Barroso S, Munoz SR, Sperduto RD, Ellwein LB. Refractive error study in children: results from La Florida, Chile. Am JOphthalmol. 2000;129(4):445-454.

8. Goh PP, Abqariyah Y, Pokharel GP, Ellwein LB. Refractive error and visual impairment in school-age children in Gombak District, Malaysia. Ophthamol. 2005;112(4):678-685.

9. He M, Zeng J, Liu Y, Xu J, Pokharel GP, Ellwein LB. Refractive error and visual impairment in urban children in Southern China. Invest Ophthalmol Vis Sci. 2004;45(3):793-799.

10. Naidoo KS, Raghunandan A, Mashige KP, et al. Refractive Error and Visual Impairment in African Children in South Africa. Invest Ophthalmol Vis Sci. 2003;44:3764-3770.

11. Pokharel GP, Negrel AD, Munoz SR, Ellewein BL. Refractive Error in Children: Result form Mechi Zone, Nepal. Am J Ophthalmol. 2000;129:43. 
12. Resnikoff S, Pascolini D, Mariotti SP, Pokharel GP. Global magnitude of visual impairment caused by uncorrected refractive errors in 2004. Bull World Health Org. 2008;86:63-70.

13. Population and Housing Census, 2000. Ghana Statistical Services., Accra.

14. Refractive Error Study in School children (RESC) Protocols and Manual of Procedures (Unpublished document).

15. El-Bayoumy BM, Saad A, Choudhury AH. Prevalence of Refractive Error and Low Vision Among School Children in Cairo. E Med Health J. 2007;13(3):575-579.

16. WHO. Global Initiative for the Elimination of Avoidable Blindness. Geneva, World Health Organization, 1989 (Unpublished document WHO.PBL/97.61).

17. Mabaso RG, Oduntan AO, Mpolokeng MBL. Refractive Status of Primary School Children in Mopani District, Limpopo Province, South Africa. S Afr Optom. 2006;65(4):125-133.

18. Adegbehingbe BO, Oladehinde MK, Majemgbasan TO, Onakpoya OH, Osagiede OE. Screening of Adolescents for Eye Disease in Nigerian High Schools. Ghana Med J. 2005;39(4):138-142.

19. Dandona R, Dandona L. Childhood Blindness in India: a population based perspective. Br J Ophthalmol. 2003;87:263-265.
20. Fotouhi A, Hashemi H, Khabazkhoob M, Mohammad K. The Prevalence of Refractive Errors among School children in Dezful, Iran. Br J Ophthalmol. 2007;91:287-292. (Available online at: http:// bjo.bmj.com/cgi/content/full/91/3/287. Accessed August 18, 2008).

21. Ovenseri-Ogbomo GO, Assien R. Refractive Error in School Children in Agona Swedru. S Afr Optom (submitted).

22. Wedner SH, Ross DA, Todd J, Anemona A, Balira R, Foster A. Myopia in Secondary School Students in Mwanza City, Tanzania: The Need for a National Screening Programme. Br J Ophthalmol. 2002;86:1200-1206. (Available online at: http://bjo.bmj.com/cgi/ content/full/86/11/1200 Accessed March 28, 2007).

23. Ho CSD, Ng CBC, Chan E, et al. Uncorrected Refractive Errors in Singapore Teenagers. Br J Ophthalmol. 2006;90:202-207. (Available online at: http://bjo.bmj.com/cgi/content/full/90/2/202. Accessed August 18, 2008).

24. Kawuma M, Mayeku R. A survey of the prevalence of refractive errors among children in lower primary schools in Kampala district. Afr Health Sci. 2002;2(2):69-72.
Clinical Optometry

\section{Publish your work in this journal}

Clinical Optometry is an international, peer-reviewed, open access journal publishing original research, basic science, clinical and epidemiological studies, reviews and evaluations on clinical optometry. All aspects of patient care are addressed within the journal as well as the practice of optometry including economic and business analyses. Basic and clinical

Submit your manuscript here: http://www.dovepress.com/clinical-optometry-journal

\section{Dovepress}

research papers are published that cover all aspects of optics, refraction and its application to the theory and practice of optometry. The manuscript management system is completely online and includes a very quick and fair peer-review system, which is all easy to use. Visit http://www.dovepress. com/testimonials.php to read real quotes from published authors. 